\title{
Potential diagnostic role of diffusion tensor imaging in early-stage osteonecrosis of the femoral head
}

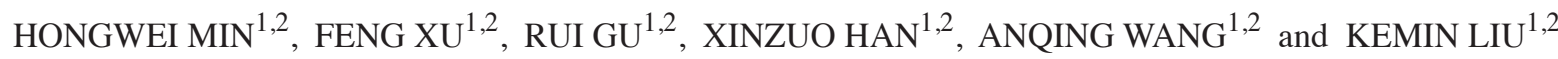 \\ ${ }^{1}$ Department of Rehabilitation, Capital Medical University; ${ }^{2}$ Department of Orthopedics and Rehabilitation, \\ China Rehabilitation Research Center, Beijing Bo'ai Hospital, Beijing 100068, P.R. China
}

Received November 14, 2014; Accepted April 8, 2016

DOI: $10.3892 /$ etm.2016.3787

\begin{abstract}
The present study aimed to explore the potential diagnostic role of diffusion tensor magnetic resonance imaging (DTI) in the early stage of modified corticosteroid-induced osteonecrosis of the femoral head (ONFH). A total of 20 beagles were randomly classified (1:1) into either an experimental group (LM), which were intramuscularly injected with lipopolysaccharide (LPS) and methylprednisolone (MPS) on three consecutive days, or control (CON) group, which were injected with saline. Magnetic resonance imaging (MRI) and DTI were performed at pre-induction and 8 and 12 weeks post-induction. Apparent diffusion coefficient (ADC) values in the range of interest in the femoral head were quantified using DTI. Proximal femora were examined for ONFH at 8 and 12 weeks. The results demonstrated that ONFH developed in four beagles at 8 weeks and in six beagles at 12 weeks, whereas no ONFH was detected in the CON group. No abnormalities were detected by MRI and DTI, and no mortality occurred. In beagles with ONFH in the LM group, the ADC values were $4.7 \pm 0.2 \times 10^{-4}$ and $4.8 \pm 0.3 \times 10^{-4} \mathrm{~mm}^{2} / \mathrm{sec}$ at 8 and 12 weeks, respectively, which were significantly increased compared with the CON group $\left(2.5 \pm 0.3 \times 10^{-4}\right.$ and $2.4 \pm 0.3 \times 10^{-4} \mathrm{~mm}^{2}$, respectively) and the LM group without ONFH $\left(2.6 \pm 0.4 \times 10^{-4}\right.$ and $2.4 \pm 0.3 \times 10^{-4} \mathrm{~mm}^{2}$, respectively) $(\mathrm{P}<0.05)$. The results of the present study indicated that intramuscular injection of LPS and MPS may lead to early-stage ONFH in beagles. As such, the detection of locally elevated ADC values in the femoral head may aid in the early diagnosis of ONFH.
\end{abstract}

Correspondence to: Dr Kemin Liu, Department of Orthopedics and Rehabilitation, China Rehabilitation Research Center, Beijing Bo'ai Hospital, 10 Jiaomen North Road, Beijing 100068, P.R. China E-mail: keminliu889@163.com

Key words: osteonecrosis of the femoral head, diffusion tensor magnetic resonance imaging, apparent diffusion coefficient, early stage

\section{Introduction}

Osteonecrosis (ON) of the hip, which predominantly affects young adults, often induces a series of unrelenting symptoms that culminate in hip pain and loss of function (1). It is estimated that 20,000 to 30,000 patients are diagnosed with ON annually accounting for $10 \%$ of the 25,0000 total hip arthroplasties performed annually in the United States (2). The disease has also been termed 'the coronary disease of the hip' by Chandler (3) as the disease simulates the ischemic condition in the heart. Osteonecrosis of the femoral head (ONFH) frequently progresses to a subchondral fracture of the femoral head, eventually leading to complete joint destruction. Joint-preserving surgical interventions are generally more successful at earlier stages of bone involvement compared with those after the occurrence of a subchondral fracture. Therefore, the optimal time to evaluate and treat osteonecrosis of the femoral head is prior to the occurrence of a subchondral fracture (4). Treatment algorithms for osteonecrosis of the femoral head are based on the staging of the lesion. Based on clinical and laboratory observations, there is typically a $\geq 3$ months delay between the occurrence of hormone-related ONFH and abnormal magnetic resonance imaging (MRI) results $(5,6)$, which is defined as the 0 -stage of the Association Research Circulation Osseous or Steinberg classification systems (7).

To date, there is no noninvasive technique to accurately diagnose 0 -stage ONFH. If early stage ONFH was diagnosed with noninvasive techniques, extracorporeal shock wave or autologous bone marrow stem cell transplantation may be used to limit the progression of ONFH, which would be of great clinical value (8). In recent years, MRI technology has developed rapidly, and the emergence of functional MRI (fMRI) has facilitated the elucidation of novel information and an in-depth understanding of various bone diseases (9), including craniofacial fibrous dysplasia, bone marrow abnormalities in multiple myeloma, systemic sclerosis, sclerosing osteomyelitis as well as metastasis of different cancers to the bone (10).

The present study aimed to explore the potential diagnostic role of diffusion tensor magnetic resonance imaging (DTI) in the early stages of ONFH in an animal model. The results of the present study may provide a theoretical basis for the clinical application of DTI for the early diagnosis of ONFH. 


\section{Materials and methods}

Animals, grouping and treatment. A total of 20 male beagles (age, 12-14 months; weight, 5.5-6.5 kg) were obtained from the Animal Center of Capital Medical University (Beijing, China) and randomly classified into one of two equal group: Control (CON) group or experimental (LM) group. Beagles were tagged and housed in cages $(\mathrm{n}=1 /$ cage $)$ under standard laboratory conditions at $20^{\circ} \mathrm{C}$ (humidity, $48 \%$ ) with a 12-h dark/light cycle. A standard diet and water were provided ad libitum. All experimental procedures adhered to the recommendations outlined by the Chinese Department of Health for the Care and Use of Laboratory Animals and were approved by the Ethics Committee of the Chinese Rehabilitation Research Center at Beijing Charity Hospital (Beijing, China). Beagles in the LM group were intramuscularly injected with $10 \mu \mathrm{g} / \mathrm{kg}$ lipopolysaccharide (LPS; Sigma-Aldrich, St. Louis, MO, USA) and $20 \mathrm{mg} / \mathrm{kg}$ methylprednisolone (MPS; Pfizer, Inc., New York, NY, USA) on three consecutive days. Beagles in the CON group were injected with normal saline. Following the daily injection, the beagles were permitted free activity.

MRI protocols. MRI and DTI were performed on the bilateral proximal femora prior to drug injection and at 8 and 12 weeks following the last injection using a $1.5 \mathrm{~T}$ superconducting magnet system (Achieva; Philips Healthcare, Best, The Netherlands). Following anesthetization via initial intramuscular injection of $25 \mathrm{mg} / \mathrm{kg}$ ketamine hydrochloride and intraperitoneal maintenance with $5 \mathrm{mg} / \mathrm{kg}$ sodium pentobarbital and blood pressure monitoring, the beagles were placed in a supine position with the lower limb flexed and fixed with adhesive tape. An extremity coil was used on the target site. MRI investigations were performed using an $1.5 \mathrm{~T}$ MRI system. All magnetic resonance pulse sequences and scan parameters used in the MRI and DTI protocols are listed in Table I. Two radiologists (independent and blinded to the investigations) assessed the MRI and DTI scans to evaluate morphologic and signal alterations in the cartilage and subchondral bone of the femoral head.

To measure the apparent diffusion coefficient (ADC) of the femoral head, a circular area (diameter, 5-mm) was selected to be the region of interest (ROI). The ROI was automatically transposed onto two maps (ADC values) generated by the image analysis software (version 17.0 SPSS, Inc., Chicago, IL, USA) (Fig. 1). ADC values of the femoral head were obtained by averaging the values obtained from the three slices selected over the target site beneath the joint space in the mid-coronal T1-weighted images (Table II).

Histological examination. Five beagles in each group were sacrificed via an overdose of sodium pentobarbital at 8 and 12 weeks following the initiation of the experiment. Tissue samples were harvested from the femoral head. Specimens $(1.0-\mathrm{mm}$ thick) were cut along the coronal plane and fixed in $10 \%$ formalin solution for 2 weeks. Following treatment with $10 \%$ nitric acid and decalcification overnight, according to conventional methods (11), the specimens were stained with hematoxylin and eosin (Shanghai Xiangjian Medical Equipment Co., Ltd., Shanghai, China). ONFH was considered present when there were empty lacunae, accumulation of bone marrow cell debris, and an increase in fat cells in the bone marrow (12). Histological analysis was performed to observe the osteonecrotic changes and repair processes in the femoral head 8 and 12 weeks post-treatment. Two pathologists (independent and blinded to the investigations of the present study) examined the tissue sections according to the criteria outlined by Arlet (13). The presence of degeneration and necrosis, the disappearance of marrow cells and nuclei and hypochromasia of trabecular osteocytes were regarded as early signs of ON (13). Sporadic empty lacunae, which may have been induced by sectioning through the edge of a lacuna, were not considered as a sign of ON.

Statistical analysis. Statistical analysis was performed using SPSS 17.0 software (SPSS, Inc., Chicago, IL, USA). Data are presented as the mean \pm standard deviation. Differences between the LM and CON groups were compared using two-sided Student's t-test. $\mathrm{P}<0.05$ was considered to indicate a statistically significant difference.

\section{Results}

Histopathological findings. No changes in form, weight, abdominal distension or tapering limbs were observed in the LM or CON groups, and no mortality occurred during the experimental period. In the CON group, no pathological alterations were observed at 8 or 12 weeks post-treatment. The articular cartilage was rich in blood vessels and intramedullary hematopoietic cells, and the trabeculae were arranged neatly and clearly. Trabecular bone cells, which were surrounded by many osteoblasts, were clearly visible, whereas empty lacunae were rare (Fig. 1). In the LM group, ONFH occurred in four beagles 8 weeks after treatment, which was detected via fat cell hypertrophy, decreased marrow hematopoietic cells, trabecular bone thinning and increasing spaces, structural disorder, partial trabecular breakage and trabecular karyopyknosis of the bone. The nuclei were small, with increasing numbers of empty lacunae detected (Fig. 2). At 12 weeks post-treatment, ONFH was detected in six animals in the LM group. The bone marrow was saturated with fat cells, bone trabecular interruption and empty lacunae; increased trabecular bone necrosis was detected around small osteoblasts, and necrotic inflammatory cells were visible. There were minimal nascent blood vessels and necrosis was detected around the areas of fibrovascular tissue repair (Fig. 3). These results demonstrate that corticosteroid treatment results in the development of ONFH in $60 \%$ of the animals.

Radiographical findings. T1-weighted images showed a high signal and T2-weighted images showed a low or intermediate signal for beagles in the CON group. No abnormal signal alterations were observed in the femoral head via MRI or DTI in any of the animals in the CON group at 8 and 12 weeks post-treatment (Fig. 4). In the LM group, the results of MRI and DTI were consistent with the CON group (Fig. 5). In the LM group, mean ADC values in the ROI of beagles with ONFH were significantly increased, as compared with the LM group without ONFH or the CON group $(\mathrm{P}<0.05$; Table II). These results further confirm that corticosteroid treatment leads to ONFH. 
Table I. Sequences and scan parameters used for magnetic resonance imaging and DTI.

\begin{tabular}{|c|c|c|c|c|c|c|c|}
\hline Purpose & $\begin{array}{c}\text { Pulse } \\
\text { sequence }\end{array}$ & $\begin{array}{l}\text { TE/TR } \\
(\mathrm{mm})\end{array}$ & $\begin{array}{l}\text { FOV } \\
(\mathrm{mm})\end{array}$ & Matrix & $\begin{array}{l}\text { Thickness } \\
(\mathrm{mm})\end{array}$ & NEX & $\begin{array}{c}\text { Specific } \\
\text { parameters }\end{array}$ \\
\hline \multirow[t]{2}{*}{ Anatomical sequence } & TSE/coronal & $35 / 520$ & $160 \times 160$ & $256 \times 256$ & 4 & 1 & \\
\hline & TSE//coronal & $100 / 2000$ & $160 \times 160$ & $256 \times 256$ & 4 & 1 & \\
\hline DTI protocol & SE.EPI & $60 / 2100$ & $160 \times 160$ & $256 \times 256$ & 4 & 4 & $\begin{array}{l}\text { VOI: } 25 \times 25 \times 2.5 \mathrm{~mm} \\
\text { b-value: } 500 \mathrm{sec} / \mathrm{mm}\end{array}$ \\
\hline
\end{tabular}

DTI, diffusion tensor magnetic resonance imaging; TE, echo time; TR, repetition time; FOV, field of view; matrix, image matrix size; NEX, number of excitations; TSE, turbo spin echo; VOI, volume of interest; SE, spin echo.
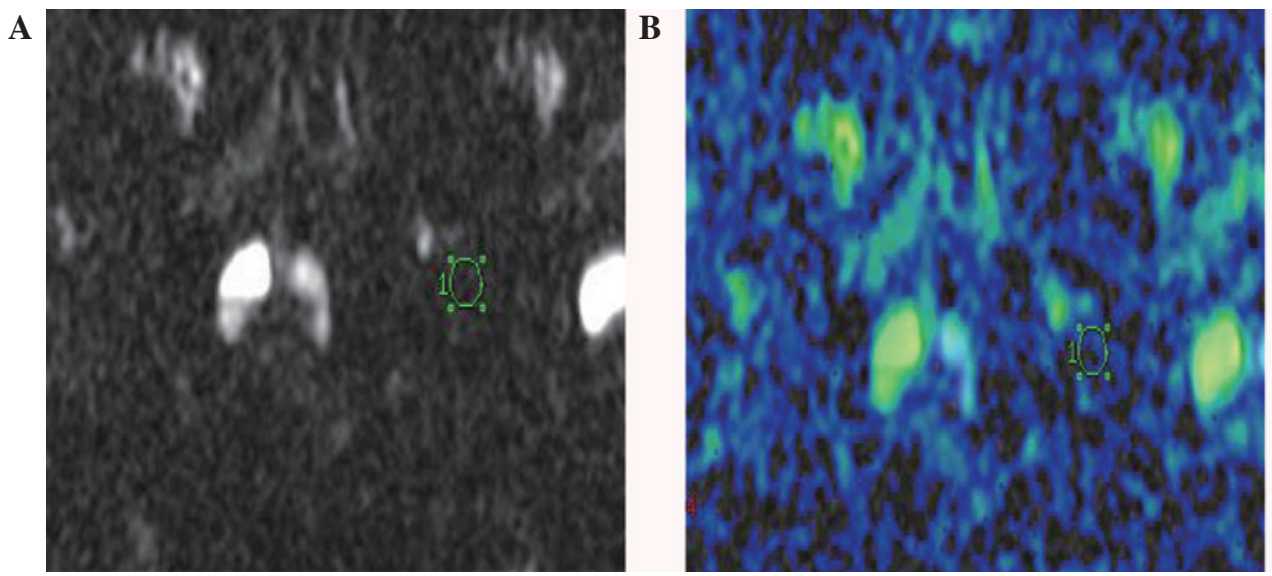

Figure 1. Diffusion tensor magnetic resonance imaging of the region of interest in the femoral head. (A) Diffusion tensor magnetic resonance imaging of the femoral head. (B) The DTI image after computer software processing in order to calculate the ADC value.

A

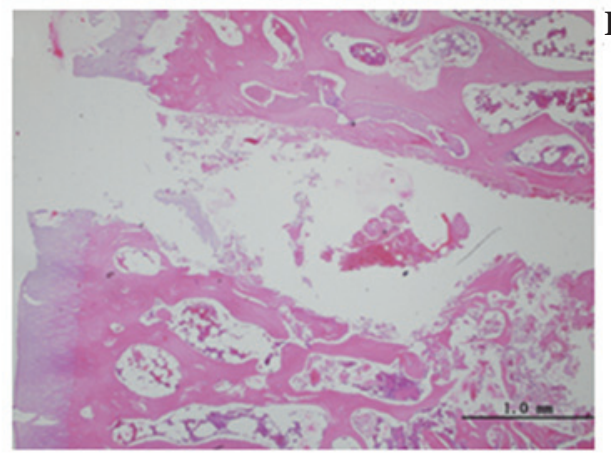

B

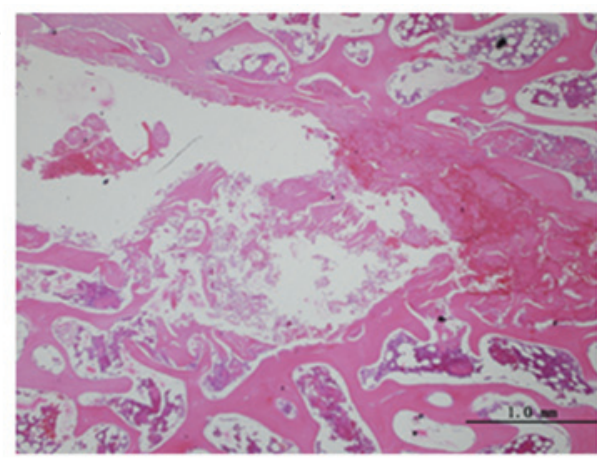

Figure 2. (A) Osteonecrosis in the subchondral zone (B) Broken trabecular bone and bone matrix dissolution in the experimental group (magnification, x40).

A

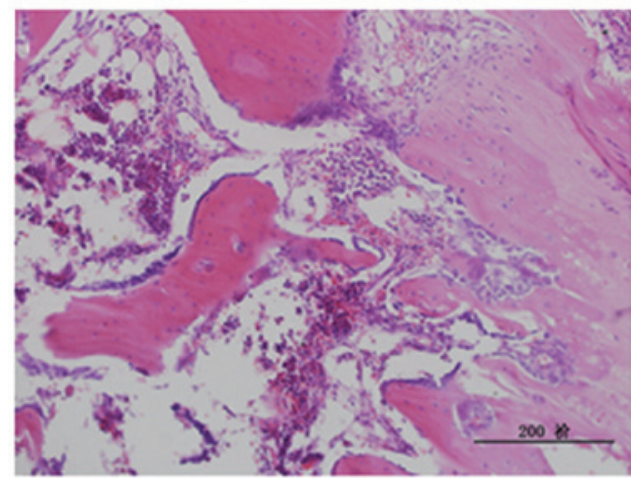

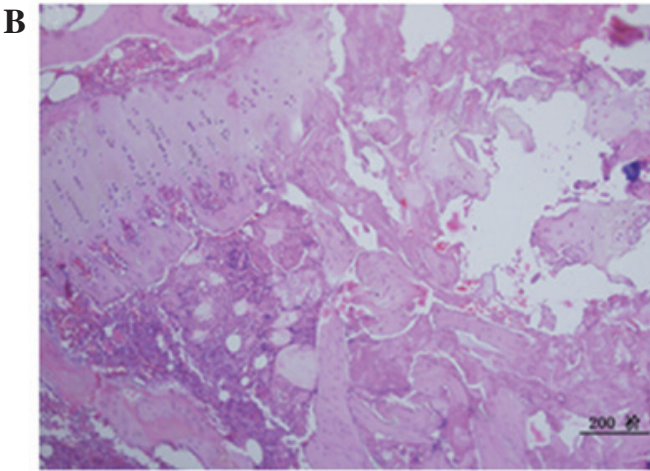

Figure 3. (A) Osteoblasts, osteoclasts (magnification, $\mathrm{x} 40$ ) and (B) fibrous tissue repair (magnification, $\mathrm{x} 100$ ) were detected in the experimental group. 
Table II. Apparent diffusion coefficient values in the proximal femur $\left(\times 10^{-4} \mathrm{~mm}^{2} / \mathrm{sec}\right)$.

\begin{tabular}{lccr}
\hline Animal group & $\begin{array}{c}\text { Prior to } \\
\text { administration }\end{array}$ & $\begin{array}{c}8 \text { weeks post- } \\
\text { administration }\end{array}$ & $\begin{array}{c}\text { 12 weeks post- } \\
\text { administration }\end{array}$ \\
\hline Beagle with ON of LM group & $2.7 \pm 0.3$ & $4.7 \pm 0.2^{\mathrm{a}}$ & $4.8 \pm 0.3^{\mathrm{a}}$ \\
Beagle without ON of LM group & $2.5 \pm 0.4^{\mathrm{b}}$ & $2.6 \pm 0.4^{\mathrm{a}, \mathrm{b}}$ & $2.4 \pm 0.3^{\mathrm{a}, \mathrm{b}}$ \\
Control group & $2.6 \pm 0.3^{\mathrm{b}}$ & $2.5 \pm 0.3^{\mathrm{a}, \mathrm{b}}$ & $2.4 \pm 0.3^{\mathrm{a}, \mathrm{b}}$ \\
\hline
\end{tabular}

${ }^{\mathrm{a}} \mathrm{P}>0.05$ vs. pre-administration in LM group; ${ }^{\mathrm{b}} \mathrm{P}<0.05$ vs. control group post-administration.
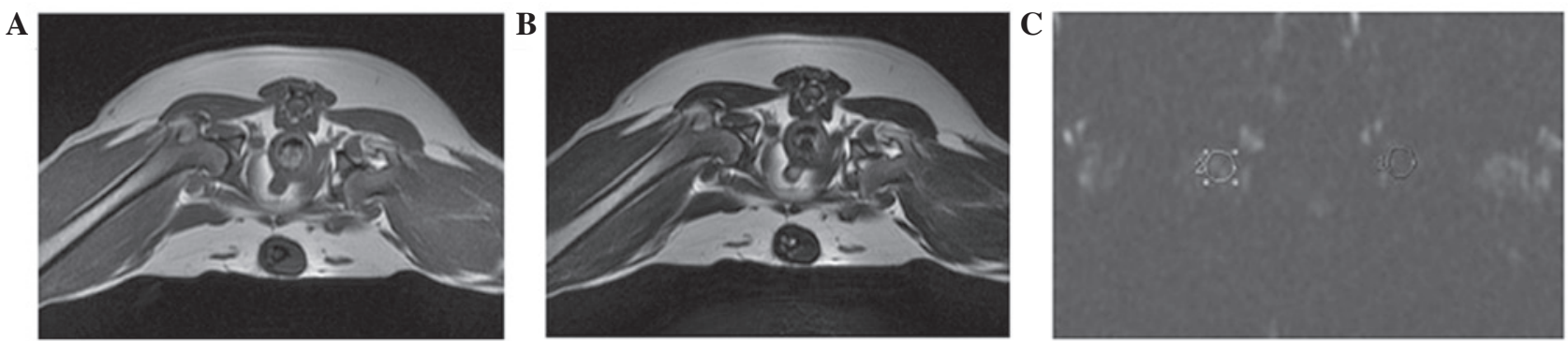

Figure 4. (A) T1- and (B) T2-weighted, and (C) diffusion tensor magnetic resonance imaging demonstrated no abnormal signal changes in the femoral head of beagles in the control group, which were treated with saline.

A

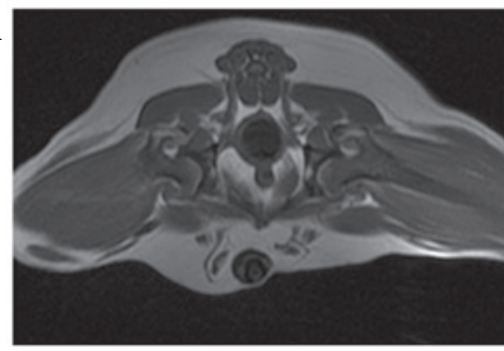

B

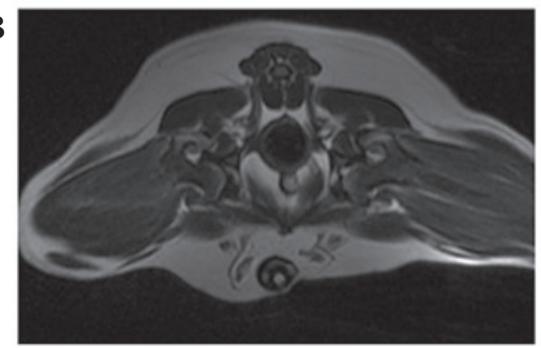

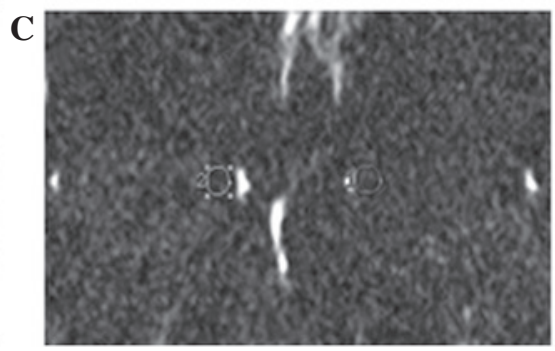

Figure 5. (A) T1- and (B) T2-weighted, and (C) diffusion tensor magnetic resonance imaging demonstrated no abnormal signal changes in the femoral head of beagles in the experimental group, which were intramuscularly injected with lipopolysaccharide and methylprednisolone for three consecutive days.

\section{Discussion}

Since the etiology of ON remains unknown, there is no standard method for the establishment of an animal model for experimental studies. Corticosteroid medication is a pivotal risk factor for the development of ON (14). Previous clinical and experimental studies have confirmed that long- or short-term intermittent use of high-dose corticosteroids may induce ON (15), and the response of both humans and animals to glucocorticoids is consistent (16).

Climcher and Kenzora (17) determined that steroids do not cause ONFH unless the patient has a disease that predisposes them to ONFH. The characteristics of $\mathrm{ON}$ induced by horse serum or endotoxin combined with steroids are similar to those of clinical ON, and are more stable than those induced by corticosteroids alone. In previous studies, a combination of low-dose LPS and high-dose MPS was used to induce $\mathrm{ON}$, resulting in a high rate of $\mathrm{ON}$ and low animal mortality $(6.2 \%)(18-23)$. Steroid injection combined with intravenous injection of LPS $24 \mathrm{~h}$ later has been demonstrated to induce a local and systemic Shwartzman reaction (shw-r), which is characterized by disseminated intravascular coagulation and results in blood coagulation disorders, blood clotting and microthrombosis formation (24).

It has previously been demonstrated that shw-r is capable of causing vasculitis, endothelial cell necrosis, hemorrhage and thrombosis, which result in tissue damage (25). As the mortality of animals with shw-r is high, a modified shw-r was elicited with a single injection of LPS combined with MPS in the large animals used in the present study. Histopathological analysis was performed 8 and 12 weeks post-treatment, which demonstrated that 4 and 6 beagles, respectively, developed ONFH. No mortality occurred. These results showed that the experimental protocol of low-dose LPS and subsequent pulsed high-dose MPS injections used in the present study is an effective method for inducing $\mathrm{ON}$ in beagles, with a high incidence of ONFH and low mortality. This animal model may be useful for subsequent studies investigating imaging alterations in the early stages of ONFH.

MRI signal intensity is predominantly correlated with the hydrogen density of the scanned organ (26); however, the pathological changes detected in $\mathrm{ON}$ are mainly due to 
alterations in fat and water content. MRI, which has a high sensitivity and specificity in the diagnosis of ON, began to be used in clinical exploration of avascular necrosis in 1983 (27). In recent years, technology has advanced to the point that MRI can be used for the early diagnosis of ONFH and the determination of pathophysiological changes in the femoral head according to imaging alterations (28). Different pathological stages of avascular necrosis exhibit differing MRI variations (29). Nevertheless, it is important to note that MRI can only detect alterations after the ON has progressed to a certain degree; therefore, even if the results of MRI are normal, ON cannot be completely ruled out. Notably, Beltran et al (30) have reported on cases of biopsy-proven ON in which the MRI scans of five patients appeared normal.

fMRI, which is an imaging technology based on conventional MRI, is a novel approach for examining patients at risk of developing ONFH. Exploratory research into the early diagnosis of ONFH with $\mathrm{OMRI}$, including magnetic resonance spectroscopy, controlled perfusion MRI and diffusion MRI, have previously been reported (31-33).

DTI is an MRI technique that has been employed to microimage cartilage and skeletal muscle (34). DTI has been demonstrated to be extremely sensitive to the morphology of the tissue examined and precise to the scale of tens of microns (35). DTI has also been used to study the changes in collagen fiber orientation under mechanical compression.

DTI facilitates the noninvasive microarchitectural characterization of heterogeneous tissue. DTI parameters of ADC of water in the bone marrow can be quantified, which provides a useful tool for the noninvasive investigation of human tissues $(36,37)$. ADC values reflect the association between the direction of the diffusion of water molecules and the organizational structure (38). These results may have been induced by increased free water in the surrounding tissue caused by acute ischemia of the femoral head and cell necrosis. MRI and DTI analysis demonstrated that the local signal strength was not significantly altered in the experimental beagles with ONFH, as compared with the beagles without. This may be due to the fact that the spatial resolution of DTI remains low, and the osteonecrotic lesions and water content of bone tissue are limited.

The results of the present study had certain limitations. Firstly, the statistical analyses are limited by the relatively small sample size. Secondly, the observation period was relatively short, which may have limited the observation of consecutive pathological and radiological changes that may occur in ON. Prolonging the observation period and establishing various time points would be of value in future studies. Furthermore, DTI also has limitations, including low resolution.

In conclusion, the results of the present study demonstrated a good correlation between the histological alterations and the DTI findings. These data may enable future studies to explore the role of fMRI in the early diagnosis of ONFH. Improvements in the hardware, image signal-to-noise ratio, spatial resolution and image post-processing software of DTI may enable the application of this technique to clinical screening, particularly in patients who exhibit at high risk for ONFH and whom conventional MRI does not show any abnormalities. As negative MRI scan cannot completely rule out $\mathrm{ONFH}$, a method of diagnosing early-stage ONFH may enable the prevention or reversal of the progression of ON. Diffusion analysis with ADC may allow physicians to obtain more detailed information on the structure of cancellous tissue, changes in bone marrow composition and bone metabolism. Further investigation and hardware improvements are required to investigate the advantages of DTI for the diagnosis of ONFH.

\section{Acknowledgements}

The present study was supported by the Basic Scientific Research Foundation of China Rehabilitation Research Center (grant no. 2013C2-19).

\section{References}

1. Petrigliano FA and Lieberman JR: Osteonecrosis of the hip: Novel approach to evaluation and treatment. Clin Orthop Relat Res 465: 53-62, 2007.

2. Mankin HJ: Nontraumatic necrosis of bone (osteonecrosis). N Engl J Med 326: 1473-1479, 1992.

3. Chandler FA: Coronary disease of the hip. J Int Coll Surg 1: 34-36, 1948

4. Ficat RP: Idiopathic bone necrosis of the femoral head. Early diagnosis and treatment. J Bone Joint Surg Br 67: 3-9, 1985.

5. Chen XC, Weng J, Chen XQ, Du JZ, Zhu MP, Pan YQ and Liu M: Relationships among magnetic resonance imaging, histological findings, and IGF-I in steroid-induced osteonecrosis of the femoral head in rabbits. J Zhejiang Univ Sci B 9: 739-746, 2008.

6. Vande Berg BC, Gilon R, Malghem J, Lecouvet F, Depresseux G and Houssiau FA: Correlation between baseline femoral neck marrow status and the development of femoral head osteonecrosis in corticosteroid-treated patients: A longitudinal study by MR imaging. Eur J Radiol 58: 444-449, 2006.

7. Schmitt-Sody M, Kirchhoff C, Mayer W, Goebel M and Jansson V: Avascular necrosis of the femoral head: Inter- and intraobserver variations of Ficat and ARCO classifications. Int Orthop 32: 283-287, 2008.

8. Wang C, Peng J and Lu S: Summary of the various treatments for osteonecrosis of the femoral head by mechanism: A review. Exp Ther Med 8: 700-706, 2014.

9. Dickerson BC: Advances in functional magnetic resonance imaging: Technology and clinical applications. Neurotherapeutics 4: 360-370, 2007

10. Bhutani M, Turkbey B, Tan E, Korde N, Kwok M, Manasanch EE, Tageja N, Mailankody S, Roschewski M, Mulquin M, et al: Bone marrow abnormalities and early bone lesions in multiple myeloma and its precursor disease: A prospective study using functional and morphologic imaging. Leuk Lymphoma 57: 1114-1121, 2016.

11. Sangeetha R, Uma K and Chandavarkar V: Comparison of routine decalcification methods with microwave decalcification of bone and teeth. J Oral Maxillofac Pathol 17: 386-391, 2013.

12. Wada M, Kumagai K, Murata M, S-Yamashita Y and Shindo H: Warfarin reduces the incidence of osteonecrosis of the femoral head in spontaneously hypertensive rats. J Orthop Sci 9: 585-590, 2004.

13. Arlet J: Atraumatic necrosis of the femoral head: General report In: Bone Circulation and Vascularization in Normal and Pathological Conditions. Schoutens A, Arlet J, Gardeniers JWM, Hughes SPF (eds). Springer US, New York, NY, pp235-240, 1993.

14. Mont MA, Jones JC and Hungerford DS: Nontraumatic osteonecrosis of the femoral head: Ten years later. J Bone Joint Surg Am 88: 1117-1132, 2006.

15. Chan KL and Mok CC: Glucocorticoid-induced avascular bone necrosis: Diagnosis and management. Open Orthop J 6: 449-457, 2012

16. Cress RL: Osteonecrosis of bone. Current concept as to etiology and pathogenesis. Clin Orthop Relat Res 208: 30-39, 1986.

17. Glimcher MJ and Kenzora JE: The biology of osteonecrosis of the human femoral head and its clinical implications. III. Discussion of the etiology and genesis of the pathological sequelae; commments on treatment. Clin Orthop Relat Res 140: 273-312, 1979. 
18. Sheng HH, Zhang GG, Cheung WH, Chan CW, Wang YX, Lee KM, Wang HF, Leung KS and Qin LL: Elevated adipogenesis of marrow mesenchymal stem cells during early steroid-associated osteonecrosis development. J Orthop Surg Res 2: 15, 2007.

19. Zhang G, Sheng H, He YX, Xie XH, Wang YX, Lee KM, Yeung KW, Li ZR, He W, Griffith JF, et al: Continuous occurrence of both insufficient neovascularization and elevated vascular permeability in rabbit proximal femur during inadequate repair of steroid-associated osteonecrotic lesions. Arthritis Rheum 60 2966-2977, 2009.

20. Zhang G, Qin L, Sheng H, Yeung KW, Yeung HY, Cheung WH, Griffith J, Chan CW, Lee KM and Leung KS: Epimedium-derived phytoestrogen exert beneficial effect on preventing steroid-associated osteonecrosis in rabbits with inhibition of both thrombosis and lipid-deposition. Bone 40: 685-692, 2007.

21. Wu X, Yang S, Duan D, Zhang Y and Wang J: Experimental osteonecrosis induced by a combination of low-dose lipopolysaccharide and high-dose methylprednisolone in rabbits. Joint Bone Spine 75 : 573-578, 2008.

22. Okazaki S, Nishitani Y, Nagoya S, Kaya M, Yamashita T and Matsumoto H: Femoral head osteonecrosis can be caused by disruption of the systemic immune response via the toll-like receptor 4 signalling pathway. Rheumatology (Oxford) 48: 227-232, 2009.

23. Sun Y,Feng Y and Zhang C: The effect of bone marrow mononuclear cells on vascularization and bone regeneration in steroid-induced osteonecrosis of the femoral head. Joint Bone Spine 76: 685-690, 2009.

24. Brozma JP: Schwartzman reaction. Semin Thromb Hemost 16: 326-332, 1990

25. Scholzen TE, Sunderkotter C, Kalden DH, Brzoska T, Fastrich M, Fisbeck T, Armstrong CA, Ansel JC and Luger TA: Alpha-melanocyte stimulating hormone prevents lipopolysaccharide-induced vasculitis by down-regulating endothelial cell adhesion molecule expression. Endocrinology 144: 360-370, 2003.

26. Milne $S$ and King GG: Advanced imaging in COPD: Insights into pulmonary pathophysiology. J Thorac Dis 6: 1570-1585, 2014.

27. Plenk H Jr, Gstettner M, Grosschmidt K, Breitenseher M, Urban M and Hofmann S: Magnetic resonance imaging and histology of repair in femoral head osteonecrosis. Clin Orthop Relat Res 386: $42-53,2001$

28. Fordyce MJ and Solomon L: Early detection of avascular necrosis of the femoral head by MRI. J Bone Joint Surg Br 75: 365-367, 1993.
29. Hauzeur JP, Sintzoff S Jr, Applelboom T, De Maertelaer V, Bentin J and Pasteels JL: Relationship between magnetic resonance imaging and histologic findings by bone biopsy in nontraumatic osteonecrosis of the femoral head. J Rheumatol 19: 385-392, 1992.

30. Beltran J, Herman LJ, Burk JM, Zuelzer WA, Clark RN, Lucas JG, Weiss LD and Yang A: Femoral head avascular necrosis: MR imaging with clinical-pathologic and radionuclide correlation. Radiology 166: 215-220, 1988

31. Hou CH, Shih TT, Liu CY, Li YD and Enright T: Proton MR spectroscopy of the femoral head-evaluation of patients at risk for avascular necrosis. J Magn Reson Imaging 24: 409-417, 2006.

32. Du J, Lu A, Dempsey M, Herring JA and Kim HK: MR perfusion index as a quantitative method of evaluating epiphyseal perfusion in legg-calve-perthes disease and correlation with short-term radiographic outcome: A preliminary study. J Pediatr Orthop 33: 707-713, 2013.

33. Yoo WJ, Kim YJ, Menezes NM, Cheon JE and Jaramillo D: Diffusion-weighted MRI reveals epiphyseal and metaphyseal abnormalities in legg-calvé-perthes disease: A pilot study. Clin Orthop Relat Res 469: 2881-2888, 2011.

34. de Visser SK, Crawford RW and Pope JM: Structural adaptations in compressed articular cartilage measured by diffusion tensor imaging. Osteoarthritis Cartilage 16: 83-89, 2008.

35. Hagmann P, Thiran JP, Jonasson L, Vandergheynst P, Clarke S, Maeder P and Meuli R: DTI mapping of human brain connectivity: Statistical fibre tracking and virtual dissection. Neuroimage 19: 545-554, 2003.

36. Chen J, Liu W, Zhang H, Lacy L, Yang X, Song SK, Wickline SA and $\mathrm{Yu} X$ : Regional ventricular wall thickening reflects changes in cardiac fiber and sheet structure during contraction: Quantification with diffusion tensor MRI. Am J Physiol Heart Circ Physiol 289: H1898-H1907, 2005.

37. Raya JG, Horng A, Dietrich O,Krasnokutsky S, Beltran LS, Storey P, Reiser MF, Recht MP, Sodickson DK and Glaser C: Articular cartilage: In vivo diffusion-tensor imaging. Radiology 262: 550-559, 2012.

38. Provenzale JM, Isaacson J, Chen S, Stinnett S and Liu C: Correlation of apparent diffusion coefficient and fractional anisotropy values in the developing infant brain. AJR Am J Roentgenol 195: W456-62, 2010. 